\title{
The Application of Reverse-Time Migration in the Z Area of PRM Basin
}

\author{
Run He ${ }^{1 *}$, Yuan Fang2, Yanchun Wang1, Lifang Cheng1, Xi Zheng ${ }^{3}$ \\ ${ }^{1}$ School of Geophysics and Information Technology, China University of Geosciences, Beijing, China \\ ${ }^{2}$ Development of Geological and Research Center, China Geological Surveys, Beijing, China \\ ${ }^{3}$ Northwest Branch Institute, Research Institute of Petroleum Exploration and Development, CNPC, Lanzhou, China \\ Email: *herun@cugb.edu.cn
}

Received 17 May 2016; accepted 26 June 2016; published 29 June 2016

Copyright (C) 2016 by authors and Scientific Research Publishing Inc.

This work is licensed under the Creative Commons Attribution International License (CC BY).

http://creativecommons.org/licenses/by/4.0/

c) (i) Open Access

\begin{abstract}
Reverse time migration (RTM) is an accurate migration method, which is based on two-way wave equation, eliminates angle limitation, and can be capable for imaging of reverse branch and multiple. In the development of an oilfield in Pearl River Mouth (PRM) Basin, RTM has been applied to solve the problems of fault shadow and structure in distinction and the drilling has proven that the RTM result is reliable. This paper introduces the theory of RTM and emphatically discusses parameter selection. The results of RTM showed that it had advantages in resolving fault shadow and the imaging of steep dip structure, and could be utilized in the oilfield.
\end{abstract}

\section{Keywords}

RTM, Wave Field Continuation, Fault Shadow, Steep Dip Structure

\section{Introduction}

At the present stage of seismic prospecting, the most widespread pre-stack depth migration (PSDM) method is the Kirchhoff integration migration based on ray theory and the wave equation migration based on one-way wave equation. The Kirchhoff PSDM is an efficient and practical technique, and possesses the features of high offset angle, no dispersion, less resource occupancy and high efficiency. However, since the application of high frequency approximation and single travel time hypothesis, the Kirchhoff PSDM is not suitable for the imaging of complex structure or drastic variable velocity condition. The one-way wave equation PSDM can resolve the complex wave in complex media and multipath problem, and has the advantage of amplitude preservation. Whereas, this method utilizes the one-way wave approximation and the simplified quasi differential equation,

${ }^{*}$ Corresponding author.

How to cite this paper: He, R., Fang, Y., Wang, Y.C., Cheng, L.F. and Zheng, X. (2016) The Application of Reverse-Time Migration in the Z Area of PRM Basin. International Journal of Geosciences, 7, 785-791. 
there are certain problems in the precise imaging of the steep-dip interface. The RTM based on two-way equation is equipped with the advantages of the above-mentioned two methods, adopts the source and detector wave field continued by full acoustic wave equation, avoids the separation of upgoing and downgoing wave, less approximates wave equation, as a consequence, overcomes the limitation of migration dip angle and migration aperture, and is appropriate for steep-dip imaging and arbitrary velocity-variation medium.

Whitmore [1] earliest proposed the RTM concept in the 53rd SEG annual meeting. At that time, due to the limitation of the computing capability in computer, RTM developed slowly. In recent year, with the rapid development of computer technology, RTM received more attention, and acquired much improvement and perfection by many scholars. Since the research area is a complex fault block oilfield and develops reservoir-controlling fracture, therefore, routine PSDM cannot satisfy the requirement of the steep-dip imaging and RTM has been adopted.

\section{Fundamental of RTM}

RTM is a depth migration method which is on the base of wave equation. RTM applies full acoustic wave equation by simulating two-way wave equation and using appropriate imaging condition to acquire image points. It does not possess the insufficient of losing correct amplitude information in integration migration and wide-angle imaging in one-way wave migration. RTM is one of the most precise migration methods in the current.

RTM had been primitively proposed by Whitmore [2] in the $53^{\text {rd }}$ SEG annual meeting in Dallas, America. Afterwards, numerous international and domestic academics do the research into it in different respects. The reverse-time propagation theory had been raised by Whitmore in the early eighties, and its basic idea is that: What happens in space completely depends on time. Although time is not reversible in physics, the time of the wave equation is reversible in mathematics. This means that the process of wave propagation in space can be observed along the time and the reverse time.

On account of the above thought, reverse-time extrapolation dose not calculate along depth, instead along time to solve the acoustic or elastic wave equation. Therefore, the known conditions are the wave field value recorded in the receiver points. For 2D seismic, the recorded wave field value is $\mathrm{P}(\mathrm{x}, \mathrm{z}=0, \mathrm{t})$; meanwhile, when $\mathrm{t}>\mathrm{T}$ ( $\mathrm{T}$ is the maximum record time of receiver points), assuming $\mathrm{P}(\mathrm{x}, \mathrm{z}, \mathrm{t}=0)$. The detailed steps of $\mathrm{RTM}$ are that defining the wave field when $t=T$ the initial value, extrapolating along the direction of the time decreased, calculating each the wave field $\mathrm{P}(\mathrm{x}, \mathrm{z}, \mathrm{t}-\Delta \mathrm{t})$ by the step size of $\Delta \mathrm{t}$. Until the wave field, when $\mathrm{t}=0$, has been calculated.

After RTM, the 3D seismic result P(x, z, t) has been obtained. Through applying appropriate imaging condition and stacking each shot record, the migration sections would be gained, as shown in Figure 1.

Imaging condition application is the critical step in RTM. Appropriate imaging condition can effectively image, even cancel noise. The widespread imaging condition at present contains excitation moment imaging condition, cross-correlation imaging condition and deconvolution imaging condition [3]. Among them, the advantage
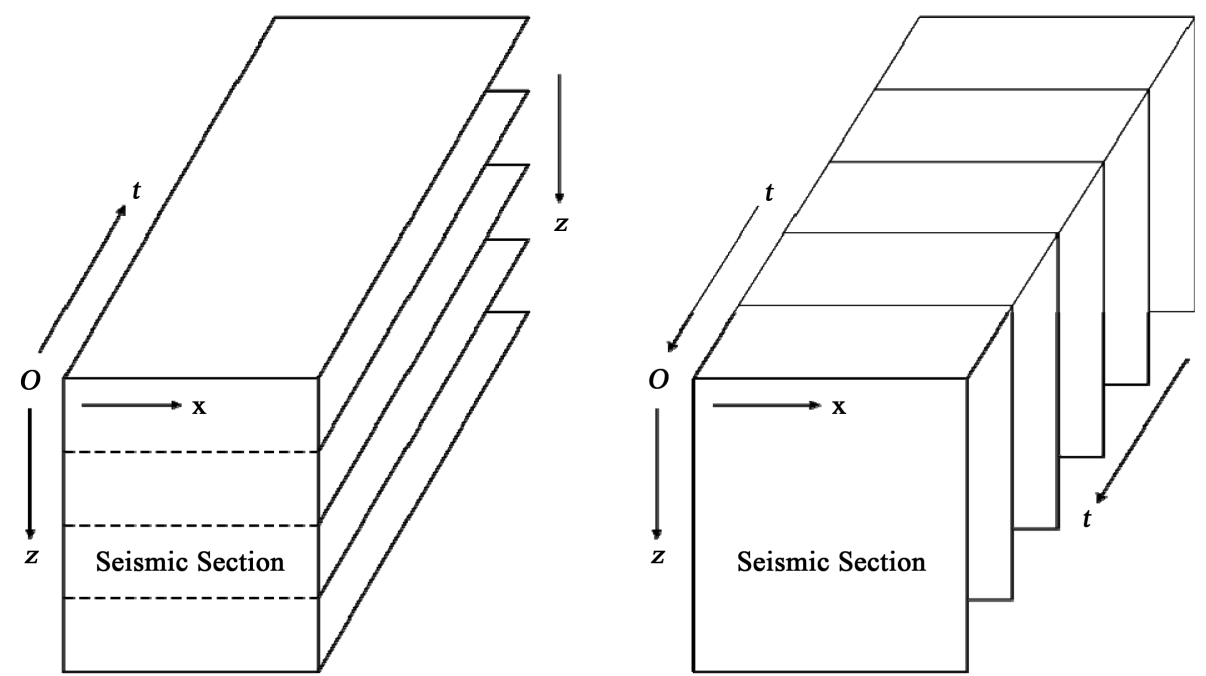

Figure 1. The schematic diagram of depth continuation (left) and reverse-time continuation (right). 
of excitation moment imaging condition is the small storage space and its disadvantage is the limitation of multipath propagation and feeblish flexibility [4]; the application of cross-correlation imaging condition is most popular, and it possesses the advantage of less computation and low calculating cost, but it would bring low frequency noise and requires higher de-noising technique [5]; the advantage of deconvolution imaging condition is high resolution and low frequency noise avoiding, but it intensely depends on wave field information and cannot guard against the interference of noise [6].

In conclusion, the concrete realization steps of RTM is divided into following four stages:

1) Forward calculation: Forward extrapolating the wave field of shot points by two-way wave equation, calculating the wave field of the wavelet generated by shot point along timer shaft, and saving the wave field information in each sampling site;

2) Reverse-time extrapolation: Backward extrapolating the wave field of receiver points along timer shaft, and saving the wave field information in each sampling site;

3) Imaging: Taking the saved source wave field and receiver wave field, analyzing imaging by appropriate imaging condition, and then finishing the RTM process of one shot;

4) Finally, repeating the above steps in each depth point of all shots, stacking each migration result of one shot, conducting necessary noise reduction, and then finishing the RTM process of the entire work area.

\section{Profile of the Research Area}

The research area is the offshore block of a certain oilfield, located in Pear River Mouth Basin of the South China Sea, and its depth of water is about $105 \mathrm{~m}$. According to the previous research results and the drilling data, it's known that reservoir-controlling fracture develops in the work area and the region entrapment is fault trap based. Committing breakpoints and sections of faults is the crux of implementing oil reservoir, confirming oil domain and deploying well positions. Whereas, in prospecting, the problems of vague fault location and steep dip imaging are always perplexing researchers, and the inaccurate explanation often brings about faulty judgment of fault sealing model and misplaced drilling (as shown in Figure 2).

During the exploration in the oilfield, two wells, well 1 and well 2, has been drilled, and they are apart from $1.7 \mathrm{~km}$. In the process of drilling, the two wells both contain oil layers in log interpretation, but the oil testing results are not the same. In the same oil layer, there is oil and gas shows in well 1, however, there is no in well 2. Analysis suggests that the inaccuracy of fault imaging causes the uncertainty of structure, therefore, the result of well 2 became a failure. In consequence, it has positive sense for utilizing RTM; besides, this application performs the guiding effect for popularizing new technique in the oilfield.
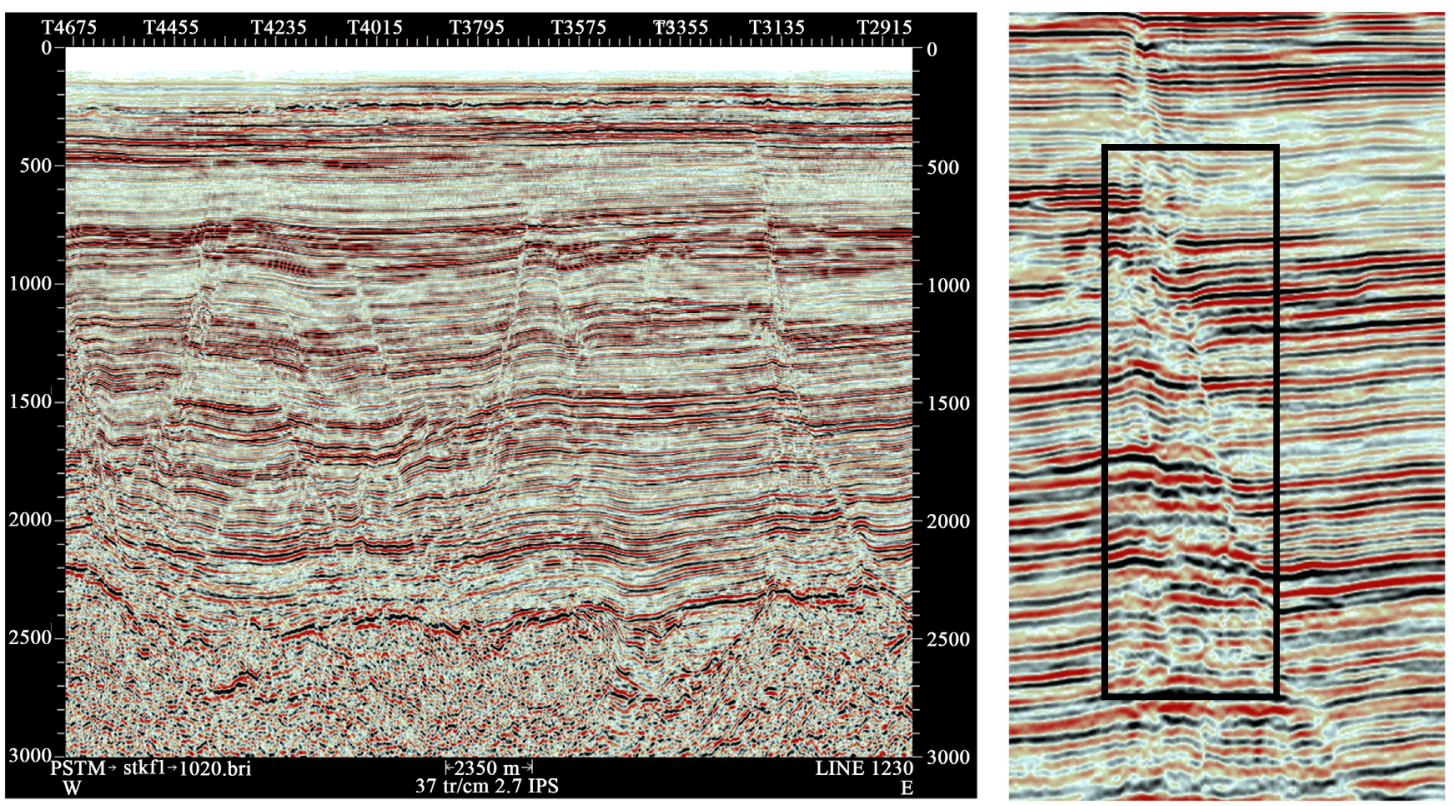

Figure 2. The schematic diagram of problems (fault shadows is in the black frame). 


\section{Migration Parameter Selection}

The selection for migration parameter plays a crucial role in RTM [7]. In the practical application, computational expense should be considered synthetically and treatment results should be optimized. In the situation of satisfying the processing demand, it's crucial to reduce calculating costs as far as possible to shorten the consuming time of mass production [8]. The primary migration parameters include velocity model, imaging condition, migration aperture, forward wavelet, continuation step, frequency band and resection angle.

Velocity model is the core factor of RTM [9]. The velocity in RTM is the same in Kirchhoff depth migration. The initial velocity model established by horizon control should be optimized and iterated in velocity by tomographic inversion, and becomes final velocity datum (Figure 3), which will be the input parameter in RTM.

The selection of imaging condition is the important step in RTM [10]. In the processing of the work area, the zero-lag cross-correlation imaging condition has been chosen. This imaging condition has the advantage of easy implementation and convenient parallel computing. In addition, the cross-correlation imaging condition is different from deconvolution imaging condition. It has no instability, will not lose wave field information, can solve the problem of multiple arrival and satisfy production needs. However, this imaging condition will introduce low-frequency noise, de-noising processing should be taken after migration imaging.

Migration aperture is the imaging space of underground information, and its size decides the seismic trace range of effective imaging points [11]. Migration aperture generally depends on the depth of purpose horizon, formation velocity and dip angle, and its area is in proportion to computing costs. Oversized and undersized migration aperture both prejudice imaging. The undersized migration aperture can insure imaging quality of simple structure and reduce computing costs, but cannot correctly image high steep structure; oversized migration aperture can obviously improve the imaging of high steep structure, but will lower integral continuity, reduce SNR and increase drastically calculating costs. Therefore, according to actual situation, migration aperture is determined by experiments [12]. In actual production, due to the too long elapsed time in RTM tests, this paper considers that migration aperture could be sure by pre-stack Kirchhoff depth migration. The migration aperture is chosen at 8000 m eventually.

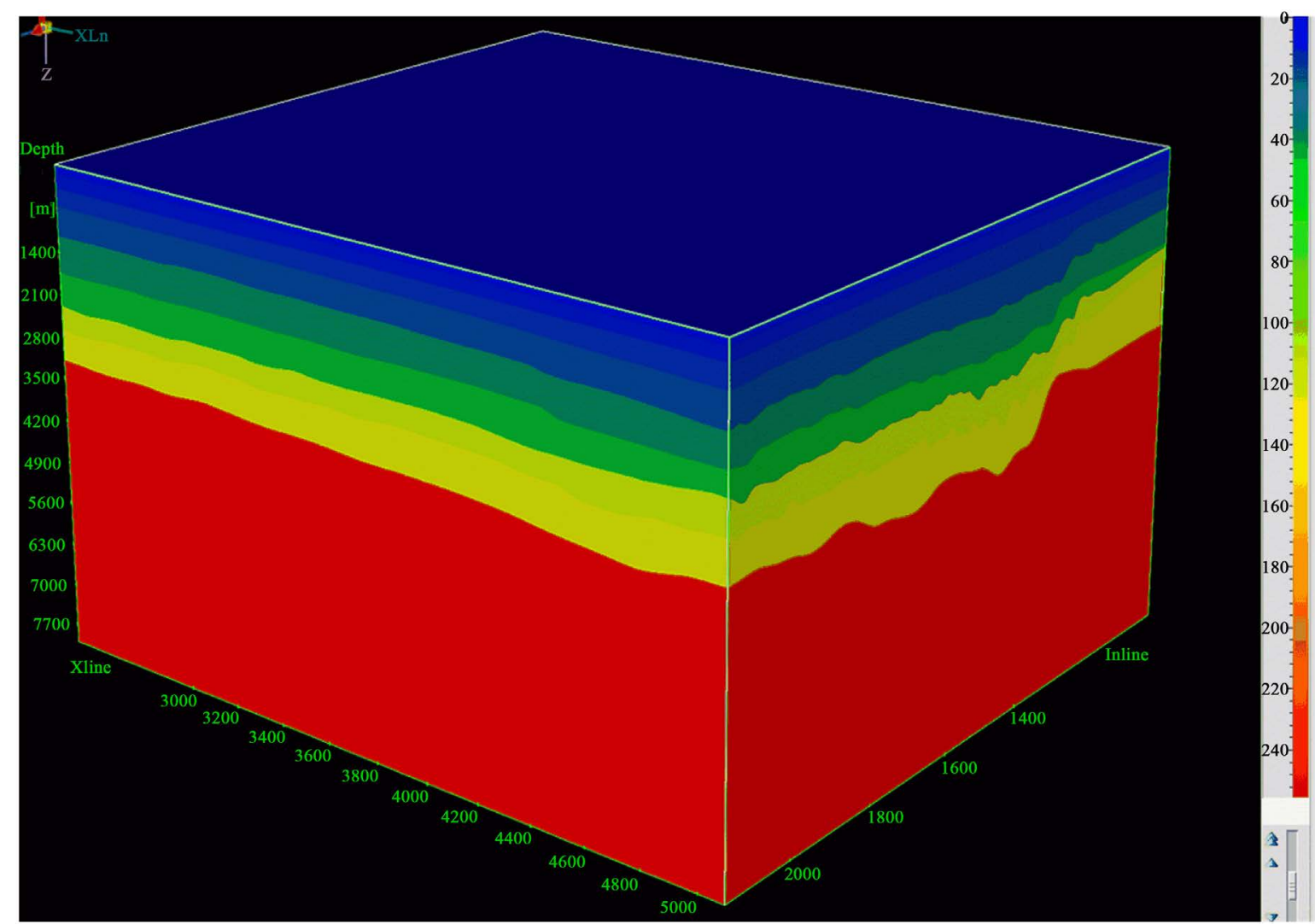

Figure 3. Initial velocity model. 
Forward continuation of seismic wave is a vital step in RTM [13]. Appropriate wavelet or not is directly relevant to the good imaging result or not. In actual production, the wavelet should be extracted from seismic data in the research area. Besides, continuation step is the key parameter for effecting computing efficiency and suitable continuation step will greatly decrease calculating costs and increase calculating efficiency. The selection of this parameter is related to the work area, dominant frequency of data, migration aperture and structural feature, thus the selection should be sure by tests.

Frequency band is the frequency domain of input data in RTM, and it's the output frequency band of RTM result. Frequency band is wider, then the frequency band of result is wider and resolution is higher, but computing costs are bigger. The selection of this parameter should consider frequency range of actual data and migration computing costs.

Due to the high costs of CRP gather in RTM, mute parameter cannot be defined on migrated CRP gather, consequently, angle mute parameter should be defined in the process of migrating. The value of this parameter is the angle of reserved data, namely, the included angle between mute line and perpendicular line.

\section{Performance of RTM}

After applying RTM in the seismic data of the work area, single-shot migration section and 3D migration stack section have been obtained. From the result of migration, the distribution of reflection layers is reasonable, waveform is well and SNR is high. The stack process of RTM is as shown in Figure 4.

Figure 5 and Figure 6 are the comparison between the results of RTM and Kirchhoff pre-stack migration in the same velocity model. It's observed that the result of RTM possesses better resolution of faults and higher SNR.

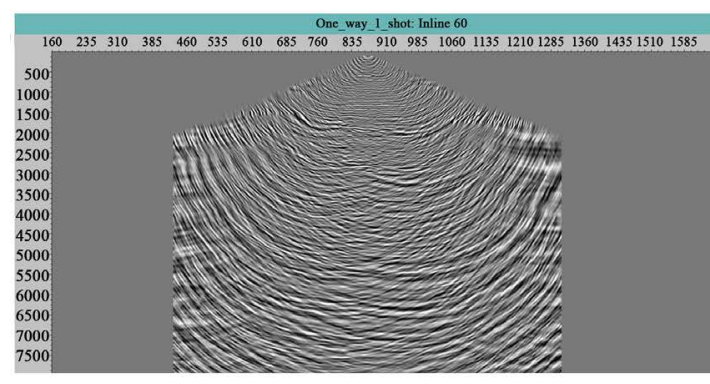

1 shot

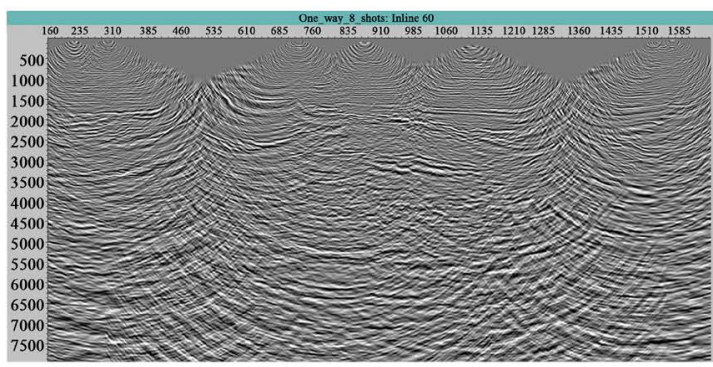

8 shot

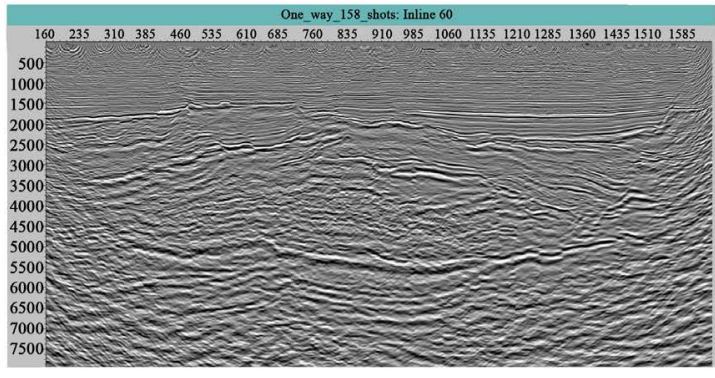

158 shot

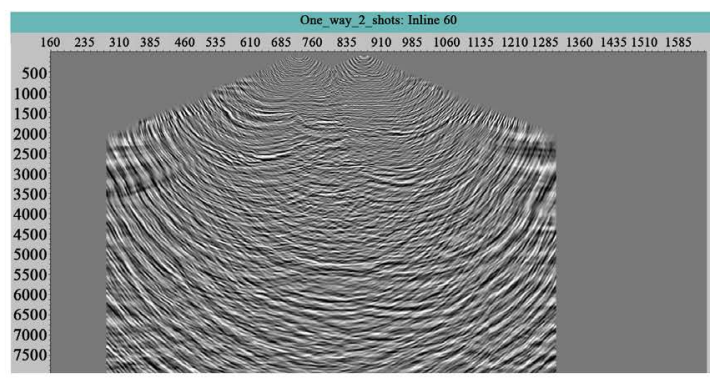

2 shot

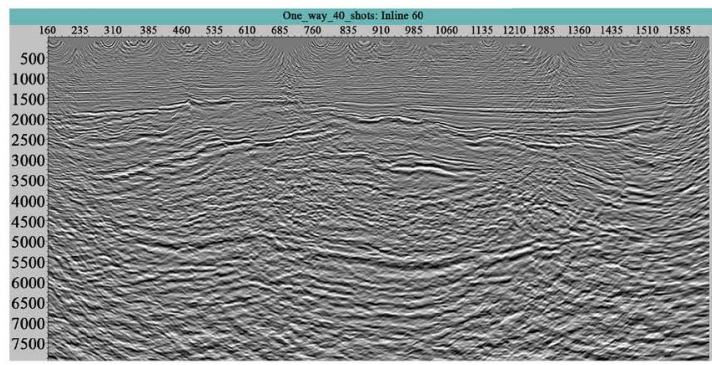

40 shot

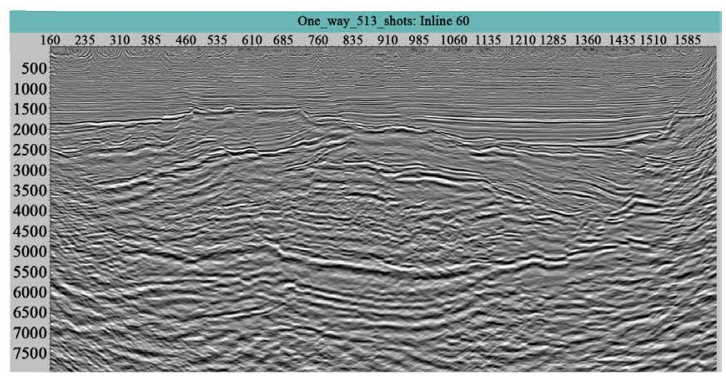

513 shot

Figure 4. The schematic diagram of RTM process. 

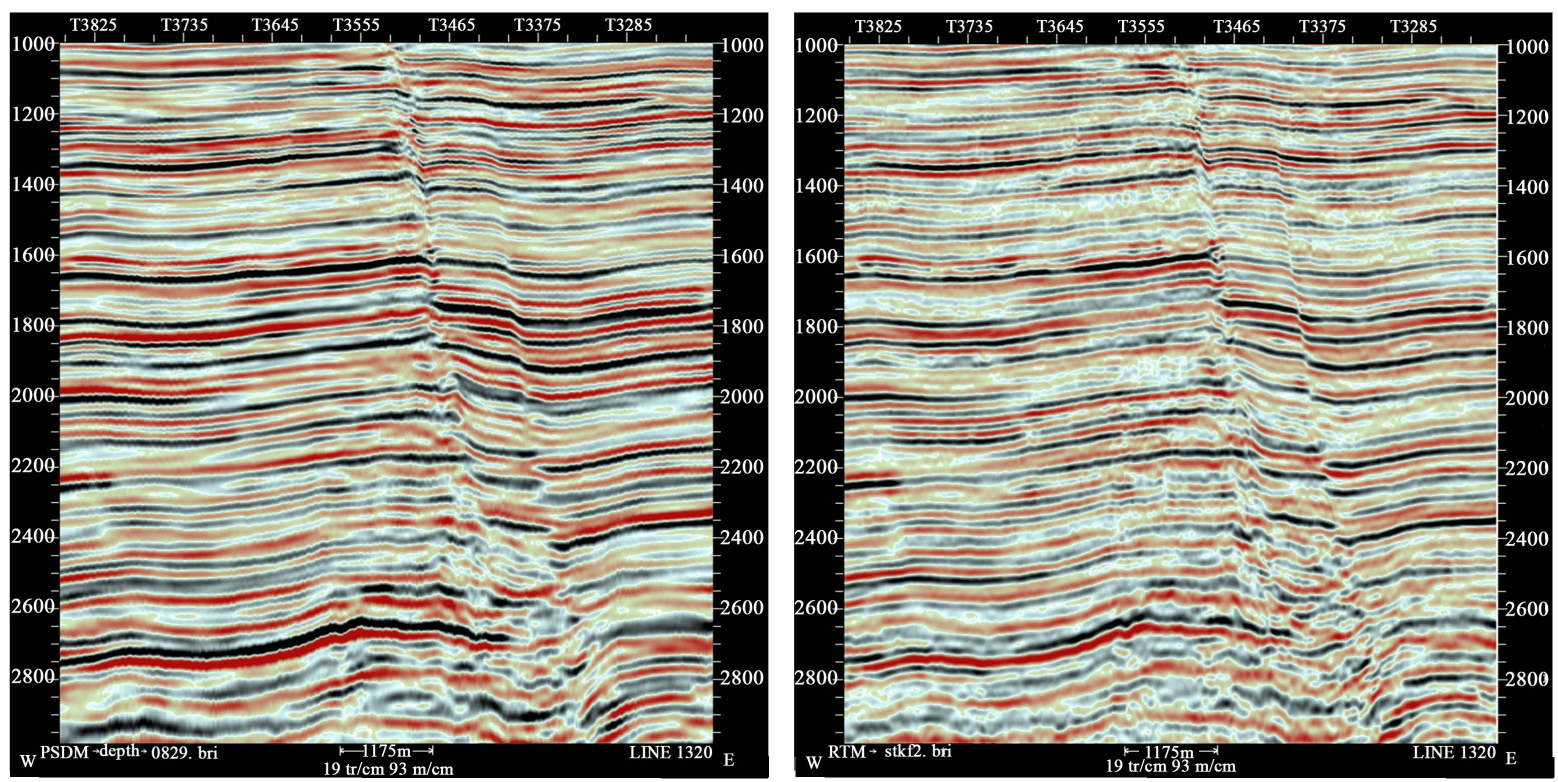

Figure 5. The analysis of Kirchhoff (left) and RTM (right) (amplification in line 1320).
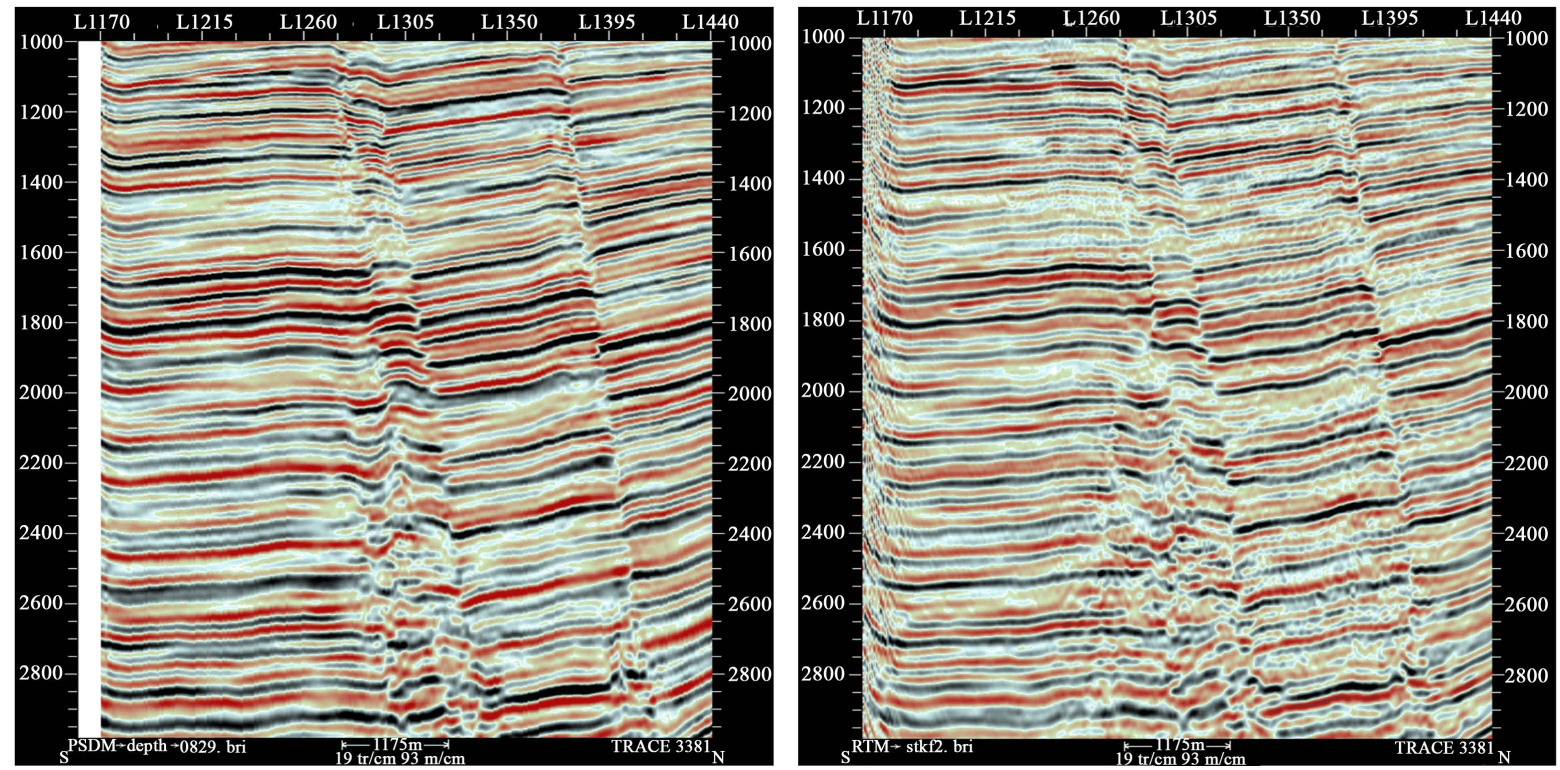

Figure 6. The analysis of Kirchhoff (left) and RTM (right) (amplification in trace 3381).

Compared with the achieved results of routine PSDM, the result of RTM has the following advantage: better continuity of reflection events in primary purpose layers, and more obvious reflection character; clean reflection background, well waveform, and stable power of wave group. The more important is that unambiguous fault imaging, clear fault points, distinct fault position and lucid occurrence features all effectively decrease the multiple solutions in interpretation, reduce the risk of drilling; the observable geologic phenomena, such as crack, pinching and overlap, is in favor of the recognition and judgment on some certain geologic phenomena, and provides good achieved data for interpreting. It should be noted that the frequency band of data has been decreased in RTM, hence, the resolution of section also has been reduced.

\section{Conclusions}

RTM is based on two-way wave equation, rids the angle limitation in one-way wave migration imaging, and can 
image reverse branch and high steep structure. The result of RTM maintains the feature of wave mechanics. RTM is the better migration imaging method at present.

The utilization of RTM in the research area has realized the imaging of high steep faults, the homing of minor fault, steady wave group power and superior imaging precision. The result of RTM precedes PSDM's. According to the verification of drilling, the processing results tally with the actual structure situation, provide strong and firm support for later-developed exploitation. The technique of RTM can be spread in high-steep structures and subsalt structure in future.

\title{
References
}

[1] Whitmore, N.D. (1983) Iterative Depth Migration by Backward Time Propagation. SEG Technical Program Expanded Abstracts, 2, 382-385.

[2] Whitmore, N.D. (1982) Migration: Fundamental Issues and Future Developments. 52nd SEG Ann. Internet. Meeting, Dallas, 22-27 September 1982, 520-520.

[3] Chattopadhyay, S. and Mcmechan, G.A. (2008) Imaging Conditions for Pre-Stack Reverse-Time Migration. Geophysics, 73, S81-S89. http://dx.doi.org/10.1190/1.2903822

[4] Jiao, Y.Y. (2011) The Research and Application of Reverse-Time Pre-Stack Depth Migration in the QT Area of Jiangsu. Master Thesis, Jilin University, Changchun. (In Chinese)

[5] Xu, L. (2012) Pre-Stack Reverse Time Migration and the Research of Removing Artifacts. Master Thesis, China University of Geosciences, Beijing. (In Chinese)

[6] Cao, Z.N. (2014) The Research and Application of Reverse-Time Migration in the TZ Area of Sinkiang. Master Thesis, China University of Geosciences, Beijing. (In Chinese)

[7] Huang, T., Zhang, Y. and Zhang, H.Z. (2009) Subsalt Imaging Using TTI Reverse Time Migration. The Leading Edge, 28, 448-452. http://dx.doi.org/10.1190/1.3112763

[8] Yang, Q.Y. and Duan, X.B. (201) Development Status and Trend of Reverse Time Migration. Geophysical Prospecting for Petroleum, No. 49, 92-98. (In Chinese)

[9] Chen, C. (2011) Pre-Stack Reverse-Time Migration and Imaging. Master Thesis, China University of Geosciences, Beijing. (In Chinese)

[10] Wei, C.L. (2013) Seismic Data Reverse-Time Migration and GPU Parallel Algorithm. Master Thesis, Ocean University of China, Tsingtao. (In Chinese)

[11] Wang, J., Li, Z.C. and Tao, L. (2012) The Research on Imaging Condition of Reverse Time Migration. Progress in Geophysics, No. 27, 1173-1182. (In Chinese)

[12] Liu, W.Q., Wang, X.W., Liu, H., Wang, Y.C., Wang, X., Zeng, H.H., et al. (2013) Application of Velocity Modeling and Reverse Time Migration to Subsalt Structure. Chinese Journal of Geophysics, No. 56, 616-625. (In Chinese)

[13] Fletcher, R., Fowler, P., Kitchenside, P. and Albertin, U. (2006) Suppressing Unwanted Internal Reflections in Pre-Stack Reverse-Time Migration. Geophysics, 71, E79-E82. http://dx.doi.org/10.1190/1.2356319

\section{Submit or recommend next manuscript to SCIRP and we will provide best service for you:}

\author{
Accepting pre-submission inquiries through Email, Facebook, Linkedin, Twitter, etc \\ A wide selection of journals (inclusive of 9 subjects, more than 200 journals) \\ Providing a 24-hour high-quality service \\ User-friendly online submission system \\ Fair and swift peer-review system \\ Efficient typesetting and proofreading procedure \\ Display of the result of downloads and visits, as well as the number of cited articles \\ Maximum dissemination of your research work
}

Submit your manuscript at: http://papersubmission.scirp.org/ 